\title{
A COMPARATIVE STUDY OF MULTIAXIAL HIGH-CYCLE FATIGUE CRITERIA APPLIED TO ROUGH ROLLING CONTACTS
}

\author{
Laure BERTHE*, Philippe SAINSOT, Antonius Adrianus LUBRECHT and Marie-Christine BAIETTO \\ Université de Lyon, CNRS \\ INSA-Lyon, LaMCoS UMR5259 \\ F-69621, France
}

\begin{abstract}
Rolling contacts are a major topic in scientific studies as they are highly exposed to fatigue damage. Indeed, the applied loads generate periodic stresses and the surface roughness generates stress concentrations close to the surface. The combination of these two phenomena facilitates fatigue damage. Many multi-axial criteria have been created to explain and predict this type of damage. Furthermore, a large number of papers compare the different fatigue criteria based on combined bending and torsion tests [1,2]. However, these studies are not representative of the stress gradients found in rough contacts, close to the surface. Hence the current work, which proposes a comparative study of several fatigue criteria with a combined experimental and numerical approach.
\end{abstract}

\section{INTRODUCTION}

The aim of this work is to test the different fatigue criteria and their applicability to a rough rolling contact. Many mutliaxial criteria are compared in the literature $[1,2]$, however, these studies are not representative of the stress gradients found in rough contacts. Moreover, the choice of the criteria, to be employed, is often difficult because of the large number of existing criteria.

A combined experimental and numerical approach is deployed [3]. Experiments were conducted on a twin-disc machine and the initiation of small cracks was detected and their propagation monitored. Numerically, different fatigue criteria are applied to a wavy Hertzian contact and to a rough Hertzian contact. The predictions are analysed for different materials based on the bending and torsional fatigue limits.

*Address all correspondence to this author.

Email: laure.berthe@insa-lyon.fr
Finally, the discs are cut up, in order to observe the crack initiation and propagation. Thus, the experimental observations indicate which criterion is most appropriate for rough rolling contacts.

\section{Experimental work}

A modified test rig, called $\mu$ Mag (Fig.1), has been used to achieve fatigue tests. This test rig allows one: (i) to perform tests one cycle at the time, (ii) to maintain the relative position of the disks during surface measurement, (iii) to measure exactly the same surface zone each time (iv) to reposition numerically the smooth and rough surfaces with respect to one other, with a precision of one micrometer. The experimental tests conducted are presented in another paper by Berthe et al. [3]
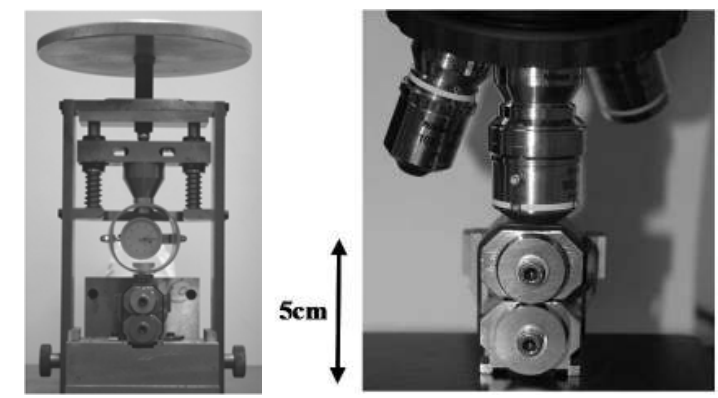

FIGURE 1. A modified twin disc machine: the $\mu \mathrm{MAG}$

The surface micro-geometry was monitored in a very detailed manner throughout the test. In fact, it allows one to detect crack initiation and the evolution of micro-pits. This also 
allows one to choose an efficient way to cut up the discs in order to observe the cracks.

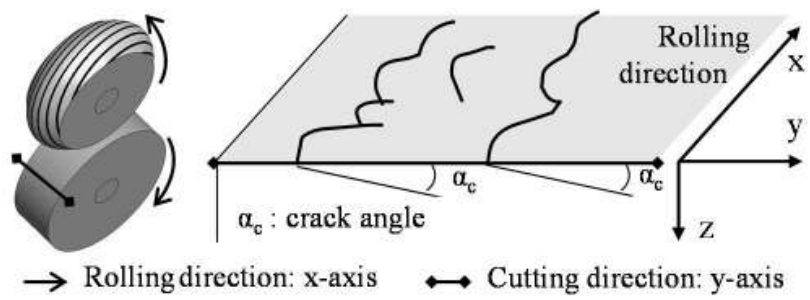

FIGURE 2. Cross-sectional view of the discs.
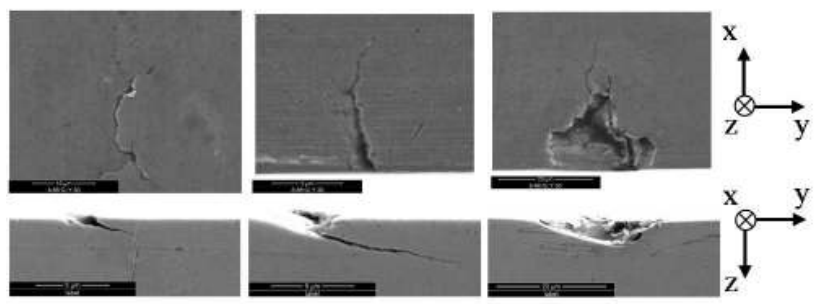

FIGURE 3. Initiation, propagation and micro-pitting observations on the smooth disc. Scanning Electron Microscope.

Figure 2 and 3 show that the micro-pitting lip formation is parallel to the rolling direction. A second observation concerns the direction of the crack development below the surface.

Furthermore, experimental results are compared with numerical rough surface fatigue results obtained with different criteria.

\section{Numerical set-up}

\section{Model}

The problem treated is a contact problem of semi-infinite bodies with a semi-analytical method. The solver is based around the conjugate gradient method and the deformation integrals are computed using Fast Fourier Transform (FFT).

In a first step, to compare the criteria, the measured surface is approximated by a wavy surface. The waviness parameters are chosen such that the contact closely matches the rough contact. One assumes that one of the bodies is perfectly smooth, the other one is covered with a waviness or with a measured roughness. The number of points is adapted to obtain a good description of asperities.

\section{High-Cycle Fatigue Criteria}

Different criteria are compared: critical plane (Dang Van [4][5], Matake [6], Findley [1][7]), stress invariants (Crossland [8]), stress averages (Papadopoulos [1]), volumetric energy (Lamefip [9][10]). All these criteria define an equivalent stress of the form:

$\sigma_{e q}=\tau+\alpha N$ where $\alpha$ is a function of $k=t_{-1} / f_{-1}$.

$t_{-1}$ is the fatigue limit in fully reversed torsion and $f_{-1}$ is the one for bending [2].

$\tau$ and $N$ are different for each criteria and represent the shear and normal stress influence respectively.

\section{Numerical results}

The wavy Hertzian contact is studied for different material parameters, following the $k$ variation from $1 / \sqrt{3}$ to 0.74 which corresponds to a large range of materials: ductile, fragile [1].

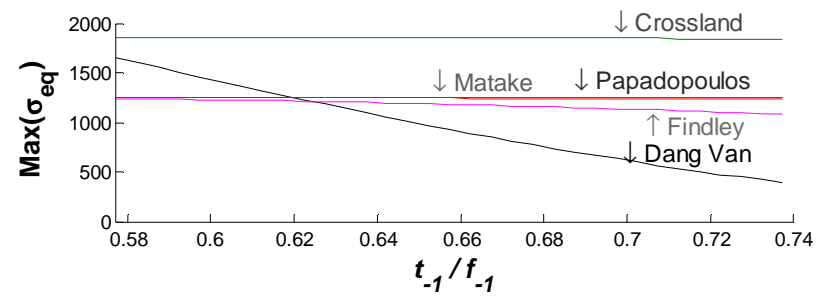

FIGURE 4. $\sigma_{\text {eq }}$ maximum for the different criteria versus $k=t_{-1} / f_{-1}$

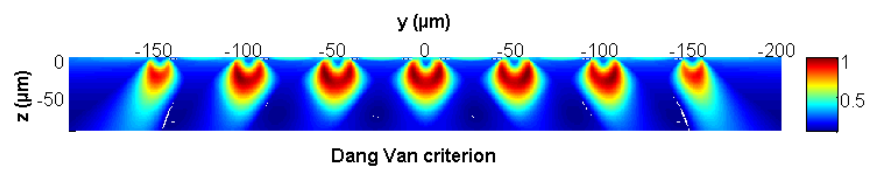

FIGURE 5. $\sigma_{\text {eq }}$ distribution using the Dang Van criterion for $k=0.68$.
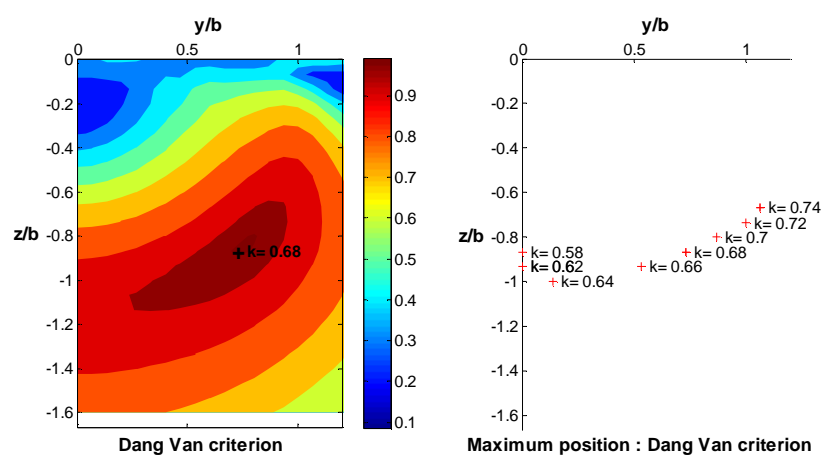

a)

b)

FIGURE 6. Stress map and maximum location using the Dang Van criterion below the central asperity ( $b$ is the half width of the asperity contact), a) $\sigma_{\mathrm{eq}}$ distribution for $k=0.68, \mathrm{~b}$ ) position of maximum $\sigma_{\mathrm{eq}}$ versus $k=t_{-1} / f_{-1}$.

All results are compared in terms of the sensitivity to the $\alpha$ parameter, which represents the relative weight of the shear and the normal stress (Fig.4). Stress maps (Fig.5-6a), maximum location (Fig.6b) and crack direction are also compared. The Dang Van criterion based on a critical plane approach predicts damage below the surface at the periphery of the asperities. The other criteria, on the contrary, predict a damage centred below the asperities. 


\section{Comparison with experimental results}

In this section, the experimental observation and numerical results are compared, like the crack direction.

The criterion that fits closest to the experimental observations is the Dang Van criterion.

For the sinusoidal roughness, the numerical results show that the Dang Van criteria is maximum on the sides of the asperities and below the surface. Experimentally, crack initiation occurs on the dotted line, see figure 7 , on either side of the grooves.

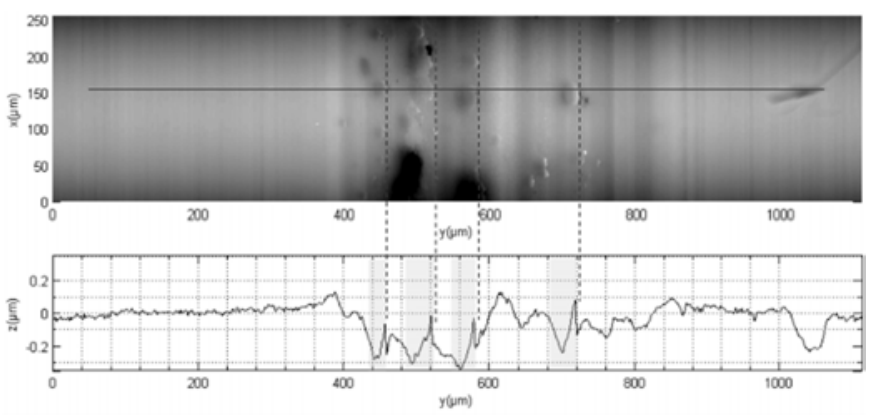

FIGURE 7. Experimental results: top figure shows topography of the smooth disc, the line indicate the position of the bottom figure roughness profile. The dotted lines show the location of crack initiation.

In addition to correctly predicting the position of the maximum stress, the Dang Van criterion gives the critical plane. In the cutting plane (Fig.8), the crack propagates at an angle of $12^{\circ}$ with the surface. Numerically, one observes an orientation of the critical plane with angles between 10 and 15 degrees.

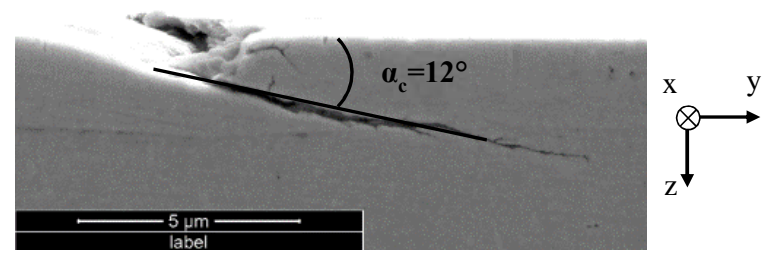

FIGURE 8. Section of the smooth disc after micro spalling. Crack propagation angle is $12^{\circ}$.

Figure 9, presents the application of the Dang Van criterion on the measured surface. The prediction show the same trends as experimentally observed.

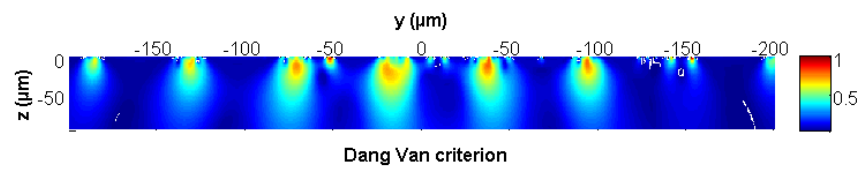

FIGURE 9. $\sigma_{\text {eq }}$ distribution of a measured rough Hertzian contact for $k=0.68$.

\section{Conclusion}

This work compared the sensitivity of different criteria for a rough rolling contact. Through a first study on a wavy Hertzian contacts, the criteria have been compared based on their severity and the location of the crack initiation. The second part of the study, the contact has been simulated using a measured rough surface. Numerical damage location and crack angles predicted are compared with the experimental damage observed. The Dang Van criterion seems the most appropriate for the current rough surface problem.

\section{REFERENCES}

[1] Papadopoulos, I. V.; Davoli, P.; Gorla, C.; Filippini, M. and Bernasconi, A., A comparative study of multiaxial high-cycle fatigue criteria for metals, International Journal of Fatigue , 1997, 19, $219-235$.

[2] Papuga, J., A survey on evaluating the fatigue limit under multiaxial loading, International Journal of Fatigue, 2011, 33, $153-165$.

[3] Berthe, L.; Sainsot, P.; Lubrecht, A. A. and Baietto, M. C., Plastic deformation of rough rolling contact: An experimental and numerical investigation, Wear, 2014, 312, 51 - 57.

[4] Dang-Van, K., Macro-micro approach in high-cycle multiaxial fatigue, ASTM Special Technical Publication, AMERICAN TECHNICAL PUBLISHERS LTD, 1993, 1191, 120-120.

[5] Sandström, J., Evaluation of Dang Van stress in Hertzian rolling contact, Fatigue \& Fracture of Engineering Materials \& Structures, Blackwell Publishing Ltd, 2012, 35, 1088 - 1094.

[6] Matake, T., An explanation on fatigue limit under combined stress, Bull JSME, 1977, 20, 257 - 263.

[7] Carpinteri, A. and Spagnoli, A., Multiaxial high-cycle fatigue criterion for hard metals, International Journal of Fatigue, 2001, 23, $135-145$.

[8] Balthazar, J. C. and Malcher, L., A review on the main approaches for determination of the multiaxial high cycle fatigue strength, Mechanics of solids in Brazil, Marcilio Alves \& Da Costa Mattos, 2007.

[9] Palin-Luc, T. and Lasserre, S., An energy based criterion for high cycle multiaxial fatigue, European Journal of Mechanics A/Solids , 1998, 17, 237 - 251.

[10] Banvillet, A.; Palin-Luc, T. and Lasserre, S., A volumetric energy based high cycle multiaxial fatigue criterion, International Journal of Fatigue, 2003, 25, 755 - 769. 